\title{
Kinetics of phase changes in large molecular clusters
}

\author{
L.S. Bartell and Th.S. Dibble \\ Department of Chemistry, University of Michigan, Ann Arbor, MI 48109, USA
}

Received 10 September 1990; accepted in final form 15 October 1990

\begin{abstract}
A method has been devised to study the kinetics of phase changes in an unfamiliar regime of extreme undercooling and rate of transformation. We show how electron diffraction can monitor the time evolution of phase changes in molecular clusters condensed from the vapor in supersonic flow. Transitions taking place in microseconds are readily followed. Examples include solid state transformations as well as the freezing of liquid clusters. Aspects of the experiment making it possible to observe familiar transitions under highly unusual conditions will be discussed along with some advantages of the new technique.
\end{abstract}

PACS: $36.40 ; 61.14 . F ;$ 64.60.Q; 82.20.M

\section{Introduction}

Structures of molecular clusters generated in supersonic flow with a carrier gas can be probed within microseconds of their formation, in situ, by electron diffraction. What is particularly interesting is that a significant degree of control over the structures of the clusters that are formed can be exercised by manipulating flow conditions. In a substantial fraction of the systems investigated it has been possible to produce clusters with either of two or, in some cases, in any of three different molecular packing arrangements (phases).

In order to learn more about the dynamics of the processes involved we recently initiated a study of the time evolution of the transformations from one cluster phase to another by probing the supersonic jet at different positions along the flow. Such monitoring yields time-of-flight resolutions adequate to characterize the kinetics of the rapid phase changes we have encountered. Under typical conditions in the flow, transitions have taken tens of microseconds for completion.

Of particular significance are (1) the unusually high degree of supercooling which can be attained, (2) the great speed of the transitions, once initiated, and (3) the derivation of interfacial free energies of boundaries between condensed phases, at least one of which is solid. In the following sections we shall briefly describe the experimental arrangement, present illustrative results, and discuss those aspects of the experimental conditions which allow observations to be made in such an unfamiliar regime of temperature and speed of transition.

\section{Experimental}

The apparatus is the University of Michigan $40 \mathrm{keV}$, photographically recording electron diffraction unit described in detail elsewhere [1]. A crucial modification making the observations feasible is the pulsed supersonic nozzle, first described in the proceedings of the ISSPIC4 symposium. A radically different, less invasive skimmer can be used, or the skimmer can be discarded altogether. This allows clusters to be monitored as soon as they leave the nozzle or subsequently, at any desired time of flight. The distance between the nozzle and the point of probing by electrons is adjusted by translating the nozzle. When clusters are produced with the $3 \mathrm{~cm}$ long Laval nozzle \#6 used in almost all prior research, one or more of the phase changes occurring may take place within the nozzle itself [2]. To postpone the changes until they can be observed, it is sometimes effective to reduce cooling rates by resorting to fairly large subject mole fractions and low stagnation pressures. A shorter Laval nozzle is often a better solution. For certain gases which form clusters easily, free jet expansion from a tubular nozzle can allow the whole sequence of cluster formation and phase changes to be monitored [3]. To grow clusters as large as those produced by Laval nozzles, it is helpful to use quite high pressures and substantially larger nozzle diameters.

Time intervals are inferred from cluster velocities measured directly under the same conditions in a separate experiment with a chopper and flight tube. Significant slippages of cluster velocities relative to gas are seen. 


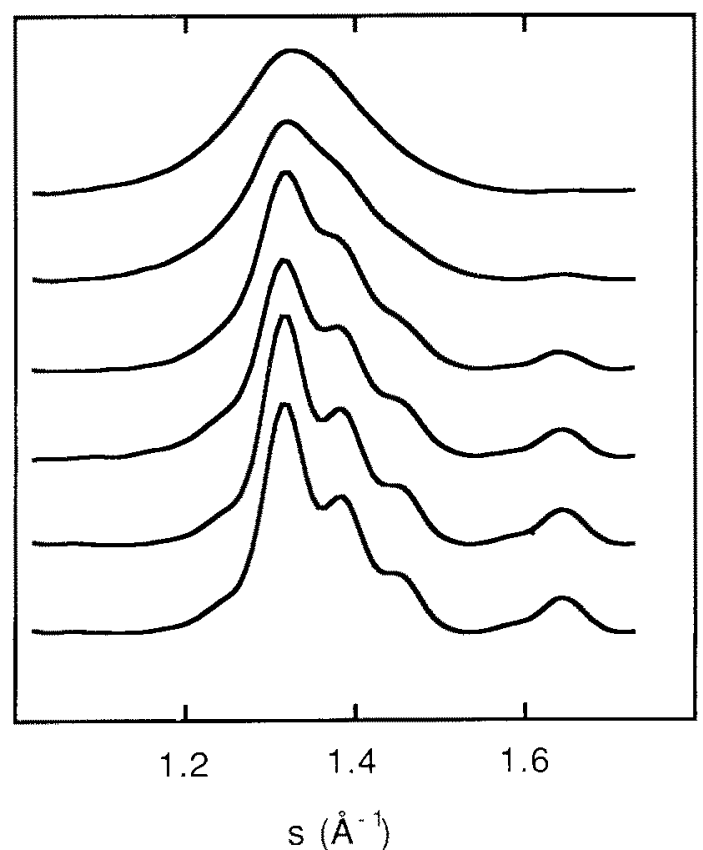

Fig. 1. Diffraction patterns of $\mathrm{CCl}_{4}$ showing freezing of liquid clusters. Time intervals between patterns, $4.5 \mu \mathrm{s}$

\section{Illustrative results}

A variety of systems undergo transitions over the conveniently observable period of a dozen microseconds or so. For the freezing of liquid clusters such systems include the chloromethyl methanes with two, three, and four chlorine atoms. For solid state transitions they include tert-butyl chloride and certain hexafluorides. Figures 1 and 2 show representative progressions of electron diffraction patterns over the course of freezing, and of solid-to-solid transformations, respectively. Cluster diameters $(12 \mathrm{~nm}$ for Fig. 1, $10 \mathrm{~nm}$ for Fig. 2) can be determined directly from the breadths of the diffraction rings. Cluster temperatures are less well known but can be estimated from modelling the nonequilibrium flow dynamics, including growth and evaporation. Transition temperatures in Figs. 1 and 2 correspond approximately to the Klots treatment of the Gspann evaporative ensemble [4].

\section{Rates of cooling and rates of transformation}

Characteristic features of the experiment set bounds on the quantities that can be observed. While this places restrictions on measurements it also opens up a heretofore unexplored regime of extreme rate of transformation associated with extreme degree of undercooling. Such exceptional undercooling can be achieved by using molecular clusters instead of the much larger particles used in previous kinetic studies of nucleation. Finely divided particles have always been advantageous in studies of the dynamics of phase change to reduce spurious effects and to keep within moderate limits the probability of forming critical nuclei during the time intervals convenient for

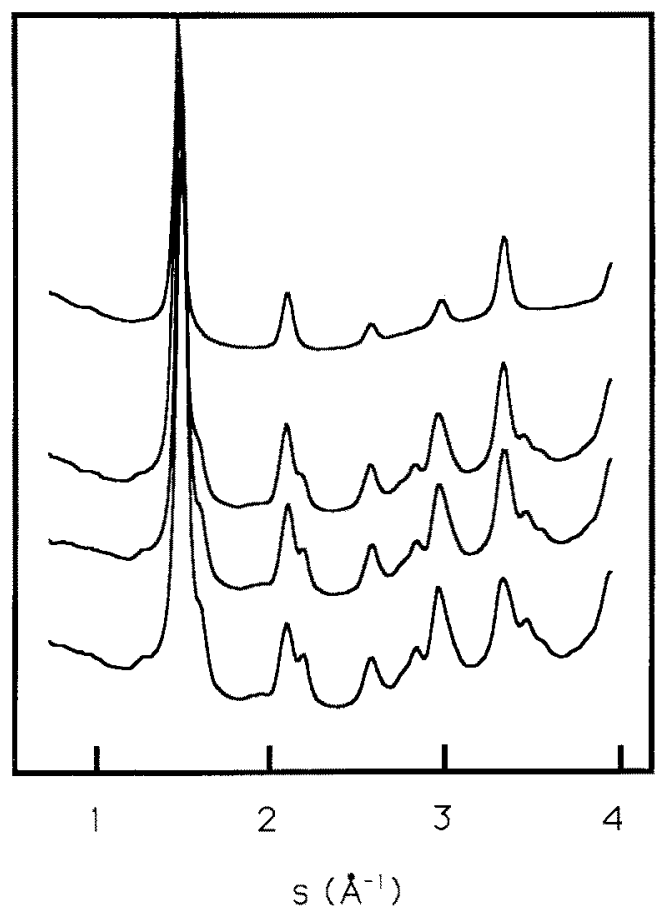

Fig. 2. Diffraction patterns of $\mathrm{SeF}_{6}$ showing a transition from bcc to monoclinic clusters. Time intervals $7.5 \mu$ s except between last two patterns where interval is $15 \mu \mathrm{s}$

measurement. Another factor in the undercooling is the rather high cooling rate characteristic of present experiments. Instead of being cooled at a constant rate of a few degrees a minute, our clusters cool by evaporation at a rate corresponding to [4]

$\mathrm{d} T=-B \mathrm{~d} \ln t$

with $B$ given approximately by $0.0015 \Delta E_{v} / R$, where $\Delta E_{v}$ represents the molar energy of evaporation. Cooling rates during transformations may be $10^{4}$ to $10^{6} \mathrm{~K} / \mathrm{s}$. Accordingly, transitions taking seconds to hours in conventional studies simply do not have time in the present work to proceed over the usual temperature range. Transitions are postponed until the driving force from undercooling accelerates the action by more than one million-fold. In many cases the rate $J$, in nuclei formed per unit volume per unit time, rises so steeply as the temperature falls that the transformation begins and ends at convenient times of flight in a supersonic jet.

If $J$ is known as a function of temperature, the fraction of clusters forming critical nuclei and transforming by time $t$ is readily calculable from the definition of $J(T)$ by integrating the expression

$\mathrm{d} N / N=J(T) V_{\mathrm{cl}} \mathrm{d} t$

where $V_{\mathrm{cl}}$ is the characteristic cluster volume. For the process of freezing the classical nucleation theory provides a feasible model and one from which a plausible temperature dependence of $J$ can be constructed if the nucleation rate is measured at one temperature. It is evident from (2) that for a system of $10 \mathrm{~nm}$ clusters to freeze in a period of, 
say, $10 \mu \mathrm{s}$, it is necessary for $J$ to be of the order of $10^{29} \mathrm{~m}^{-3} \mathrm{~s}^{-1}$. While this is many orders of magnitude higher than most rates cited in the literature, it is not uncommon for systems undercooled $30 \%$ below their transition temperature. For the system we have studied most completely, $\mathrm{CCl}_{4}$, an application of classical nucleation theory suggests that such a nucleation rate is close to the maximum that the system can attain. The free energy barrier to nucleation rises when the liquid is warmed, slowing freezing, but molecular motions become too sluggish in the viscous liquid to form critical nuclei rapidly when the system is cooled below $T_{\max }$.

It is worth noting that for substances with maximum nucleation rates in the vicinity of $10^{29} \mathrm{~m}^{-3} \mathrm{~s}^{-1}$ subjected to evaporative cooling rates, clusters much smaller than $10 \mathrm{~nm}$ in diameter have a small probability for forming nuclei as they pass through the region of $T_{\max }$. Although they may not be truly quenched into a glassy state, their freezing may be considerably delayed. Conversely, clusters much larger than $10 \mathrm{~nm}$ begin to transform at more moderate undercoolings. It is not believed that the molecular behavior in $10 \mathrm{~nm}$ clusters is significantly different from that in the bulk. Clusters of about $10 \mathrm{~nm}$ can be seen, then, to be especially convenient subjects for study. They are easy to produce and control in size, they have become just large enough to yield well-characterized electron diffraction patterns, and they allow a probing of phase transitions in an interesting regime of rate. Variations of nozzle design, flow conditions, and substance condensed make it possible to see rich variations in the kinetics of phase change. Results can be expected to lead to an increased understanding of the molecular behavior in such processes.

\section{References}

1. Bartell, L.S., Heenan, R.K., Nagashima, M.: J. Chem. Phys. 78, $234(1983)$

2. Bartell, L.S., Harsanyi, L., Valente, E.J.: J. Phys. Chem. 93, 6201 (1989)

3. Beck, R.D., Hineman, M.F., Nibler, J.W.: J. Chem. Phys. 92, 7068 (1990)

4. Gspann, J.: Physics of electronic and atomic collisions. Datz, S. (Ed.). Washington: Hemisphere 1976 